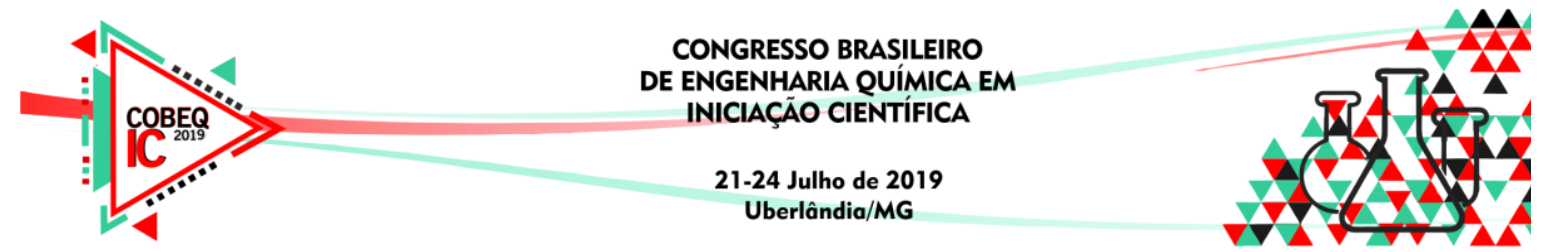

\title{
AVALIAÇÃO DO EQUILÍBRIO LÍQUIDO VAPOR DE UMA MISTURA DE ETANOL + TOLUENO À TEMPERATURA CONSTANTE UTILIZANDO SIMULAÇÃO NUMÉRICA EM MATLAB PARA MODELOS DE COMPOSIÇÕES LOCAIS
}

\author{
V. A. de ABREU ${ }^{1}$, A. T. SANTOS ${ }^{1}$, W. R. O. PIMENTEL ${ }^{1}$ e A. K. BRAGA ${ }^{1}$ \\ ${ }^{1}$ Universidade Federal de Alagoas, Centro de Tecnologia, Departamento de Engenharia \\ Química \\ E-mail para contato: valdeir4991@ gmail.com
}

\begin{abstract}
RESUMO - A variação da composição de misturas através das propriedades das substâncias é essencial quando se envolve processos de destilação, adsorção e extração líquido-líquido. Com isso, a fim de avaliar como a composição do sistema varia em função da temperatura, foi feito um estudo referente ao equilíbrio líquidovapor de uma mistura de etanol e tolueno considerando a temperatura constante de 308,15 K através de simulação numérica. Utilizou-se um algoritmo em Matlab e parâmetros disponíveis na literatura para obtenção dos coeficientes de atividade através do método de Wilson, NRTL e UNIFAC. Foram obtidas melhores aproximações em relação aos valores experimentais para o modelo UNIFAC nas regiões próximas ao ponto de azeótropo. Entretanto, em regiões afastadas do ponto de azeótropo, com pequenas frações de etanol na fase líquida, melhores aproximações foram obtidas utilizando o modelo NRTL.
\end{abstract}

\section{INTRODUÇÃO}

\subsection{Equilíbrio Líquido-vapor}

O conceito de equilíbrio é estabelecido a partir da estabilização do sistema com o decorrer do tempo, em que a taxa de transferência de moléculas de uma fase para a outra se dá de forma constante. No equilíbrio líquido-vapor há a coexistência de fases distintas e diagramas que descrevem a variação da composição com a temperatura e pressão podem ser obtidos. Estes são chamados "diagramas de fases", em que há a região de vapor superaquecido, líquido subresfriado e a região de equilíbrio líquido-vapor para sistemas ideais (baixas pressões, altas temperaturas e espécies quimicamente similares). (Smith, 2007)

Porém, em aplicações industriais, nem sempre são utilizadas espécies similares a baixas pressões, sendo necessário o trabalho com espécies que apresentam desvios da idealidade. Com isso, estudos que visem o entendimento de problemas de equilíbrio líquido-vapor, com comportamento distante da idealidade, podem ser de grande relevância. Como visto no trabalho desenvolvido por Zhu et al (2007), em que há aplicação do equilíbrio líquido vapor na destilação com pressões variáveis, sendo aplicado na indústria farmacêutica, produção de esteróides e mistura com solventes, além da adição de componentes para facilitar a separação. 


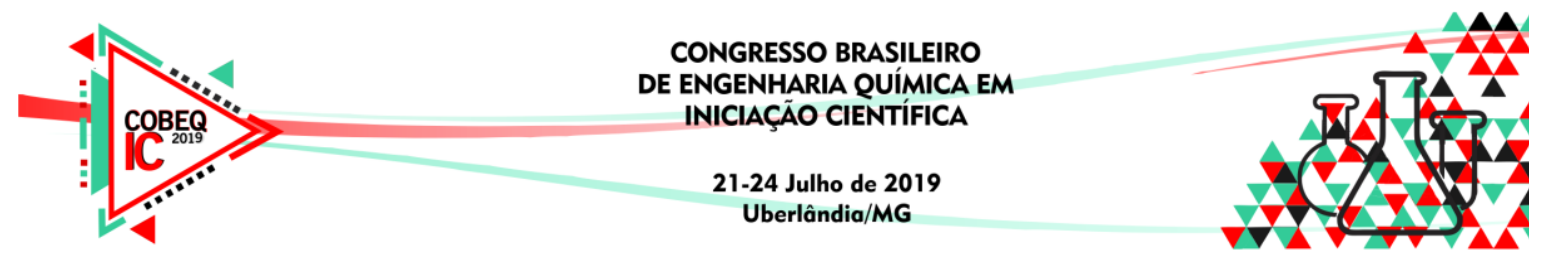

\subsection{Lei de Raoult e Coeficiente de Atividade}

A lei de Raoult prevê comportamentos (com relação à composição de misturas) assumindo que a fase vapor se comporta como gás ideal e a fase líquida tem o comportamento de solução ideal sendo possível trabalhar apenas nas condições enfatizadas anteriormente. Ela é dada conforme a Equação 1.

$$
y_{i} \cdot P=x_{i} \cdot P_{i}^{s a t}
$$

Sendo $y_{i}$ a composição na fase vapor, $x_{i}$ a composição líquida e $P_{i}^{s a t}$ a pressão de saturação do componente $i$, que pode obtido pela equação de Antoine . (Smith, 2007)

Porém quando se trata de sistemas a altas temperaturas com compostos não similares quimicamente, há um desvio da idealidade da mistura em que a Lei de Raoult não é válida. Com isso, é utilizado na equação de Raoult um coeficiente de atividade que irá variar de acordo com as naturezas das espécies, levando-se em conta os desvios da idealidade. A Lei de Raoult modificada se dá conforme a Equação 2. (Smith, 2007)

$$
P \cdot y_{i}=x_{i} \cdot \gamma_{i} \cdot P_{i}^{s a t}
$$

Em que $\gamma_{i}$ representa o coeficiente de atividade da substância $i$.

Quando a composição de um componente na fase vapor é igual à composição do mesmo componente na fase líquida, tem-se um ponto característico, denominado ponto de azeótropo. $\mathrm{Na}$ indústria química procura-se evitar este ponto, em virtude da dificuldade na realização da separação dos componentes, mesmo tendo volatilidades diferentes.

\subsection{Modelos para o Coeficiente de Atividade}

Modelo de Wilson: $\mathrm{O}$ modelo de Van Larr para o coeficiente de atividade descrevia o comportamento de misturas, porém apresentava altos desvios em regiões diluídas. Assim, em 1964 foi proposta por Wilson um conjunto de equações levando em consideração coeficientes de atividade para diluição infinita de ambas as espécies, considerando a composição local. Neste é levado em conta tanto o tamanho das moléculas quanto as interações intermoleculares. (Wilson, 1964).

Modelo NRTL: O modelo NRTL (Non-Random Two-Liquid), desenvolvido por Renon e Prausnitz (1968), assim como o modelo de Wilson, aborda a ideia de composição local. Porém, este modelo abrange a possibilidade de cálculos de equilíbrio envolvendo duas fases em estado líquido. (Renon, 1968)

Modelo UNIFAC: $\mathrm{O}$ método para determinação do coeficiente de atividade para o equilíbrio líquido-vapor, utilizando o modelo UNIFAC, está baseado nas contribuições dos grupos constituintes das moléculas presentes na mistura. Inicialmente, a avaliação das interações entre grupos distintos foi proposta por Langmuir no ano de 1925, baseado no fato de que, apesar de haver milhares de componentes químicos de interesse industrial, o número de grupos funcionais que constituem esses componentes é muito menor. Assim, as interações 


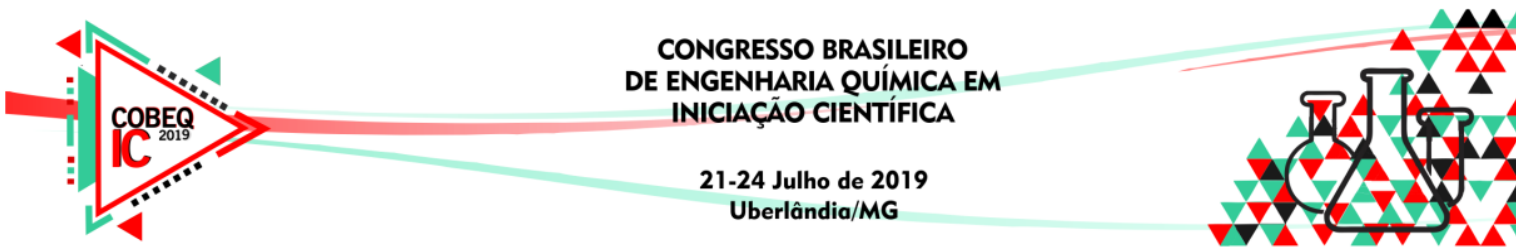

físicas de Van der Waals que ocorrem entre duas ou mais moléculas podem ser calculadas como a soma das interações entre os grupos funcionais que constituem essas moléculas. Esta é uma forma aproximada de predizer propriedades de compostos a partir do conhecimento das propriedades de seus grupos. (Schuhli, 2007)

Aplicações dos métodos de composição local foram realizadas no trabalho desenvolvido por Kwak et al (2007), em que um estudo da mistura binária de tolueno e etanol mostrou bons resultados na obtenção dos parâmetros utilizados nos modelos de Wilson, NRTL e UNIQUAC. Com isso, no presente estudo, buscou-se analisar o comportamento da mistura de etanol e tolueno, a partir de diferentes modelos de composição local, e compara-los aos dados experimentais. $\mathrm{O}$ entendimento do comportamento desta mistura é justificado pelo fato de serem um dos principais componentes das águas residuais de refinarias de petróleo.

\section{METODOLOGIA}

\subsection{Parâmetros}

Wilson e NRTL: Para que fossem utilizadas no algoritmo foram utilizados os valores disponíveis na literatura, os quais constam na Tabela 1. Os parâmetros $a_{12}, a_{21}, b_{12}, b_{21}$ possuem unidade $\mathrm{cal} / \mathrm{mol}$, e $V_{1}$ e $V_{2}$ possuem unidade de $\mathrm{cm}^{3} / \mathrm{mol}$. (Smith, 2007)

Tabela 1 - Parâmetros das equações de Wilson e NRTL.

\begin{tabular}{|c|c|c|c|c|c|c|}
\hline \multirow{2}{*}{$\begin{array}{c}\text { Componente } \\
\text { ( i ) }\end{array}$} & \multicolumn{3}{|c|}{ Equação de Wilson } & \multicolumn{4}{|c|}{ Equação NRTL } \\
\cline { 2 - 7 } & $V_{i}$ & $a_{12}$ & $a_{21}$ & $b_{12}$ & $b_{21}$ & $\alpha$ \\
\hline Etanol (1) & 58,68 & 1556,45 & 210,52 & 713,57 & 1147,86 & 0,5292 \\
\hline Tolueno (2) & 106,85 & & & & & \\
\hline
\end{tabular}

UNIFAC: Levando em consideração que os parâmetros chave do método UNIFAC são os valores de $\mathrm{R}$ e $\mathrm{Q}$ de cada grupo - Tabela 2. Os valores de $v$ são relativos às quantidades dos subgrupos em cada um dos componentes. Sendo o componente (1) o etanol e (2) o tolueno.

Tabela 2 - Parâmetros utilizados nas equações do método UNIFAC.

\begin{tabular}{|c|c|c|c|c|}
\hline Subgrupo & $\mathrm{R}_{\mathrm{i}}$ & $\mathrm{Q}_{\mathrm{i}}$ & $\mathrm{V}_{\mathrm{i}}{ }^{(1)}$ & $\mathrm{V}_{\mathrm{i}}^{(2)}$ \\
\hline $\mathrm{CH}_{3}$ & 0,9011 & 0,8480 & 1 & 0 \\
\hline $\mathrm{CH}_{2}$ & 0,6744 & 0,5400 & 1 & 0 \\
\hline $\mathrm{OH}$ & 1,0000 & 1,2000 & 1 & 0 \\
\hline $\mathrm{CH}$ (Aromático) & 0,5313 & 0,4000 & 0 & 5 \\
\hline $\mathrm{CH}_{3}$ (Ligado ao aromático) & 1,2663 & 0,968 & 0 & 1 \\
\hline
\end{tabular}

\subsection{Simulações}

Foi desenvolvido um algoritmo em Matlab, utilizando o software gratuito Octave, a fim de se calcular os coeficientes de atividade através de cada método de composição local abordado. Foi realizada a avaliação das condições da mistura de etanol + tolueno quando a 


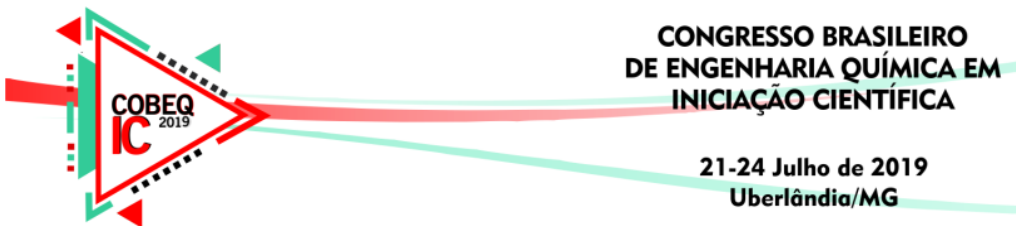

composição da fase líquida dos componentes variou de 0 a 1, utilizando a lei de Raoult Modificada - Equação 2 - para obtenção da composição da fase vapor e da pressão à temperatura constante de $303,15 \mathrm{~K}$.

Figura 1 - Algoritmo para obtenção e utilização dos coeficientes de atividade a partir de diferentes modelos de composição local na construção dos diagramas presentes nas Figuras 2 e 3.

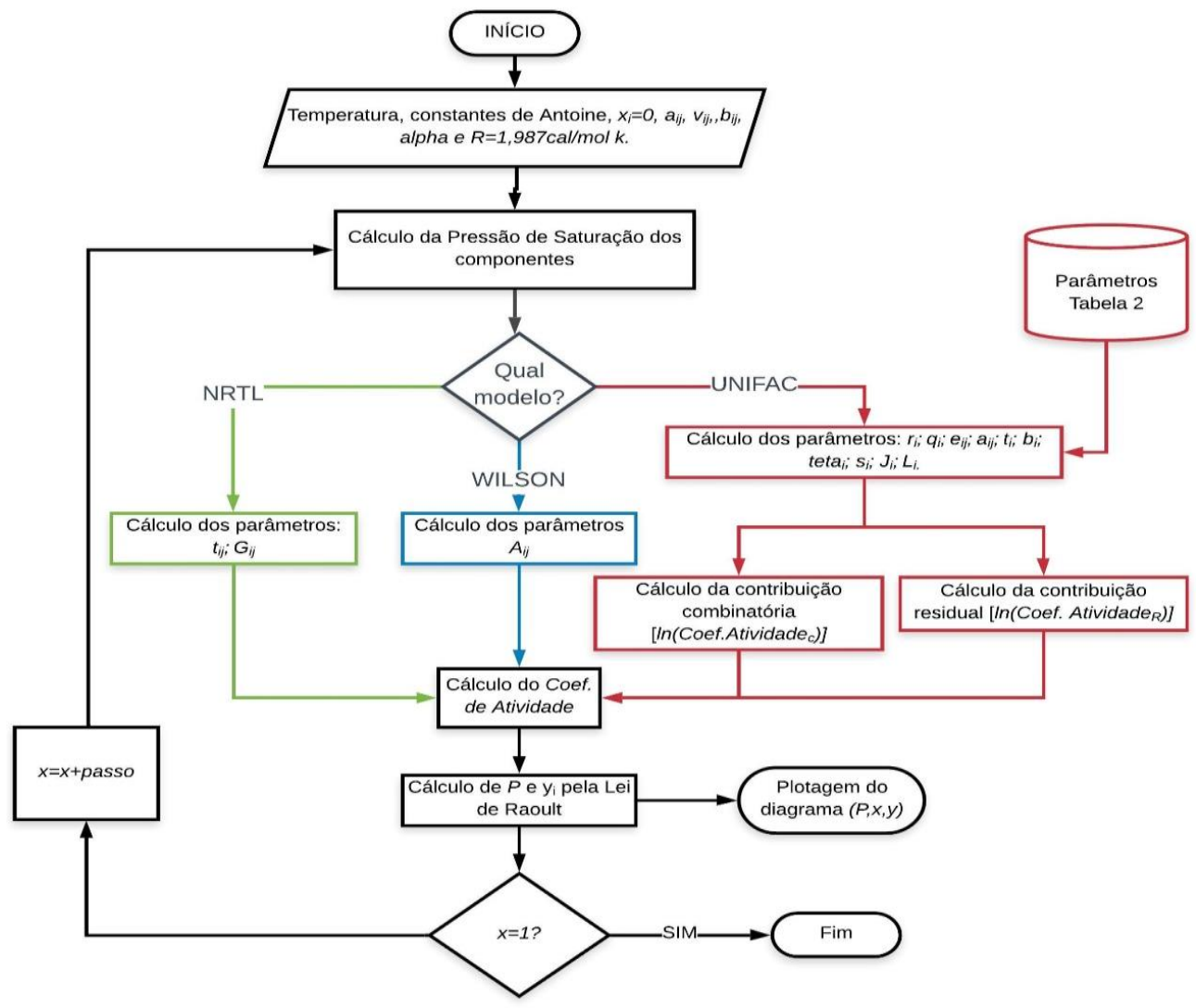

\section{RESULTADOS E DISCUSSÃO}

A partir das simulações feitas através do algoritmo desenvolvido para os métodos de Wilson, NRTL e UNIFAC para a mistura de etanol e tolueno, foi possível avaliar com base nos dados experimentais dispostos na literatura a eficácia e a abrangência (relativa à composição) dos respectivos métodos de composição local. Os valores experimentais serviram como referência para os cálculos de erro relativo percentual. Tais valores foram obtidos por Kwak (2007).

$$
\% \text { Erro relativo }=\frac{\mid \text { valor }_{\text {exp }}-\text { valor }_{\text {cal }} \mid}{\text { valor }_{\text {exp }}} * 100 \%
$$

Onde, valor $_{\text {exp }}$ corresponde aos valores experimentais e valor $_{\text {cal }}$ aos valores calculados para cada método de composição local. 


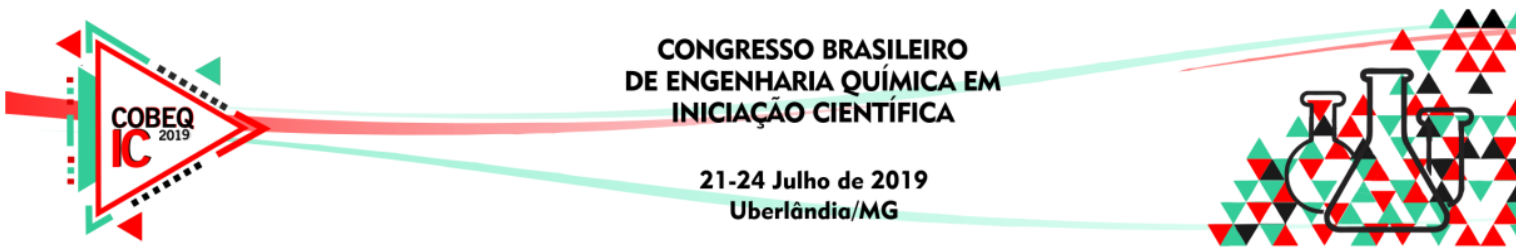

Figura 2 - Valores experimentais para os pontos de bolha $(\mathrm{X})$, para os pontos de orvalho ( ), curva Px (一) e curva Py ( - obtidas pelo método de (a) NRTL e (b) Wilson para a mistura binária de etanol + tolueno.

(a)

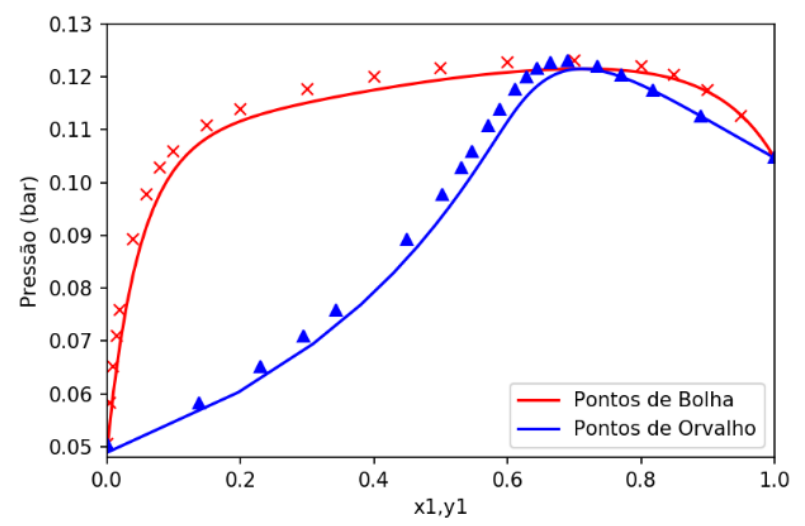

(b)

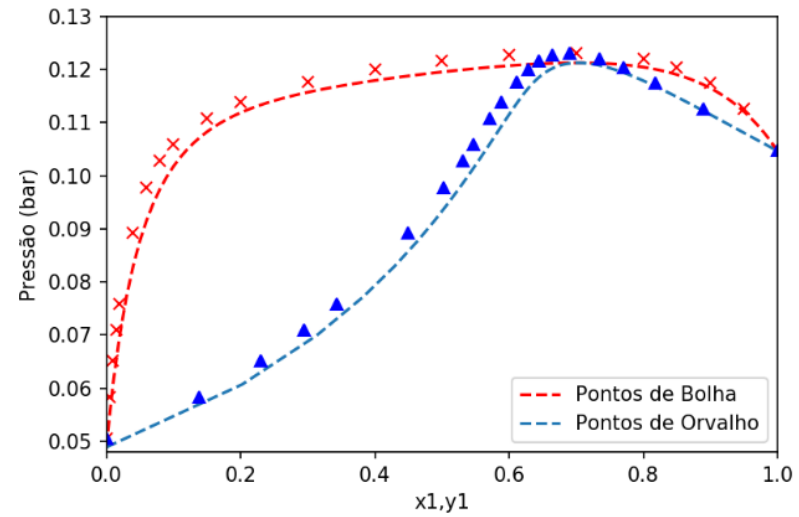

Para os modelos de Wilson e NRTL, foram obtidas boas aproximações para todas as faixas de concentrações do etanol na fase líquida, além disto, apresentaram boa aproximação na região do ponto de azeótropo $\left(x_{1}=y_{1}=0,705\right)$ - Figuras 2 a e Figuras $2 \mathrm{~b}$.

Já para o método UNIFAC, observa-se uma variação considerável nos valores de pressão em regiões diluídas, apesar disto, o referente método apresentou os menores valores de erros relativos quando avaliado na região do ponto de azeótropo - Figuras 3 e 4 .

Figura 3 - Valores experimentais e curvas dos pontos de bolha e orvalho obtidas pelo método UNIFAC para a mistura binária de etanol + tolueno.

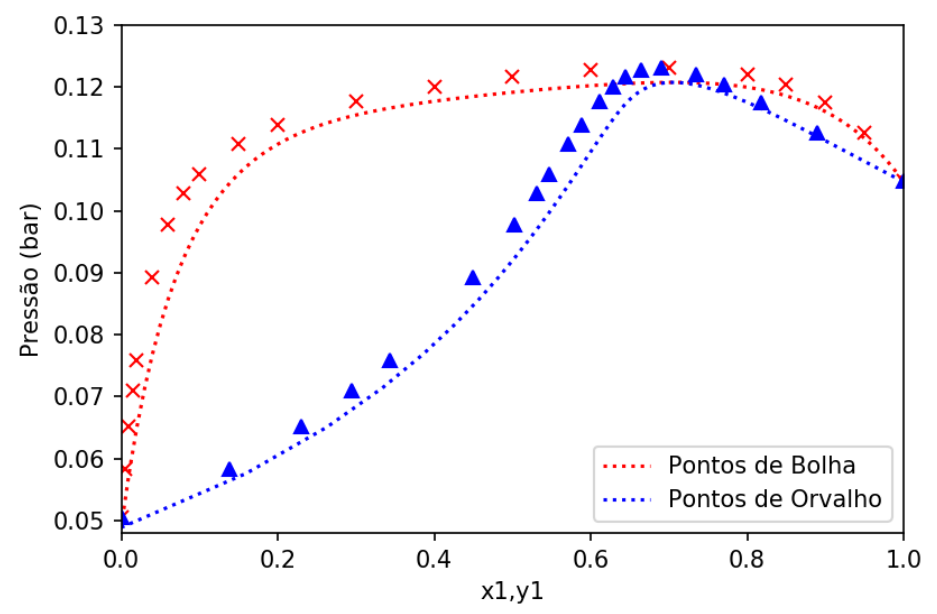

Através da análise nas Figura 1a-b e 2, em que estão dispostos o comportamento das simulações, podendo ser notado uma boa aproximação pelo método UNIFAC quando a composição se aproximava do ponto de azeótropo. Porém, em concentrações muito pequenas do etanol, os modelos Wilson e NRTL apresentaram melhores aproximações dos valores de pressão.

É possível verificar através da Figura 3, em que é mostrado os valores dos erros relativos, nas regiões próximas ao ponto de azeótropo, o método UNIFAC apresentou os menores desvios, demonstrando as observações feitas anteriormente. 


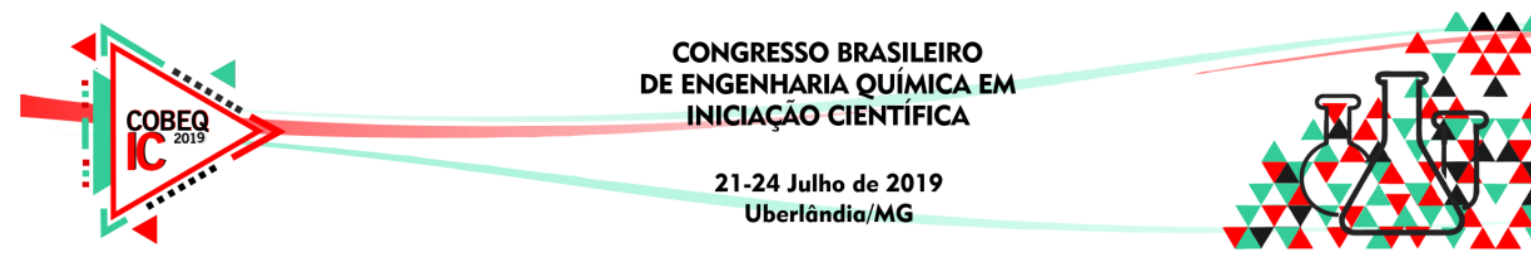

Figura 4 - Valores de erros para a fração de vapor nos métodos de NRTL, Wilson e UNIFAC.

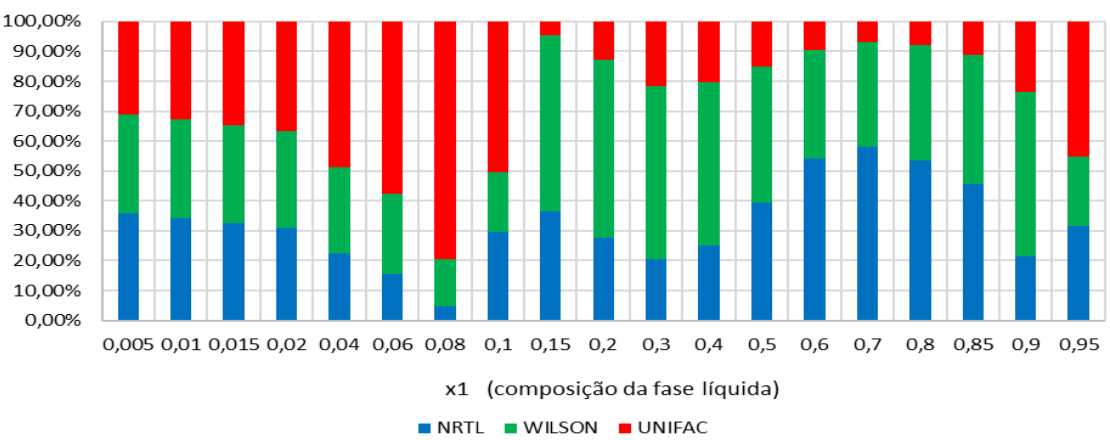

\section{CONCLUSÃO}

-NRTL WILSON U UNIFAC

Por meio das simulações feitas através de um algoritmo desenvolvido em Matlab, foi possível verificar a eficácia dos modelos de composição local de Wilson, NRTL e UNIFAC para a mistura de etanol + tolueno quando comparados aos valores obtidos na literatura. Foram obtidos valores de erro para os modelos, considerando os valores experimentais como referência. Através disso, foram observados menores valores de erro na região próxima ao ponto de azeótropo da mistura quando utilizado o método UNIFAC e maiores quando utilizados o modelo de NRTL. Portando, em regiões próximas ao ponto de azeótropo para a mistura etanol + tolueno, os valores obtidos utilizando o modelo UNIFAC apresentaram maior correlação aos dados experimentais, demonstrando a eficácia deste método que pode ser aplicado a problemas de equilíbrio líquido-vapor de âmbito industrial.

\section{REFERÊNCIAS}

$\mathrm{KOH}$, Sang-Mo; DIXON, Joe Boris. Preparation and application of organo-minerals as sorbents of phenol, benzene and toluene. Applied Clay Science, v. 18, n. 3-4, p. 111$122,2001$.

KWAK, Hae-Yeon et al. Isothermal vapor-liquid equilibrium at $333.15 \mathrm{~K}$ and excess volumes and molar refractivity deviation at $298.15 \mathrm{~K}$ for the ternary system di-butyl ether (1)+ ethanol (2)+ toluene (3) and its binary subsystems. Fluid Phase Equilibria, v. 262, n. 1-2, p. 161-168, 2007.

RENON, Henri; PRAUSNITZ, John M. Local compositions in thermodynamic excess functions for liquid mixtures. AIChE journal, v. 14, n. 1, p. 135-144, 1968.

SMITH, J. M., NESS, V. H. C. e ABBOTT, M. M. Introdução à Termodinâmica da Engenharia Química, 7a Ed., LTC - Livros Técnicos e Científicos Editora, Rio de Janeiro, 2007.

WILSON, Grant M. Vapor-liquid equilibrium. XI. A new expression for the excess free energy of mixing. Journal of the American Chemical Society, v. 86, n. 2, p. 127-130, 1964.

ZHU, Zhaoyou et al. Separating an azeotropic mixture of toluene and ethanol via heat integration pressure swing distillation. Computers \& Chemical Engineering, v. 76, p. 137-149, 2015. 